Pacific

Journal of

Mathematics

\author{
ON THE RELATIONSHIP OF \\ CONTINUITY AND BOUNDARY REGULARITY IN \\ PRESCRIBED MEAN CURVATURE DIRICHLET PROBLEMS
}

Kirk E. LANCASTER AND JARON MELIN 


\title{
ON THE RELATIONSHIP OF CONTINUITY AND BOUNDARY REGULARITY IN PRESCRIBED MEAN CURVATURE DIRICHLET PROBLEMS
}

\author{
KIRK E. LANCASTER AND JARON MELIN
}

\begin{abstract}
In 1976, Leon Simon showed that if a compact subset of the boundary of a domain is smooth and has negative mean curvature, then the nonparametric least area problem with Lipschitz continuous Dirichlet boundary data has a generalized solution which is continuous on the union of the domain and this compact subset of the boundary, even if the generalized solution does not take on the prescribed boundary data. Simon's result has been extended to boundary value problems for prescribed mean curvature equations by other authors. In this note, we construct Dirichlet problems in domains with corners and demonstrate that the variational solutions of these Dirichlet problems are discontinuous at the corner, showing that Simon's assumption of regularity of the boundary of the domain is essential.
\end{abstract}

\section{Introduction}

For $n \in \mathbb{N}$ with $n \geq 2$, suppose $\Omega$ is a bounded, open set in $\mathbb{R}^{n}$ with locally Lipschitz boundary $\partial \Omega$. Fix $H \in C^{2}\left(\mathbb{R}^{n} \times \mathbb{R}\right)$ such that $H$ is bounded and $H(x, t)$ is nondecreasing in $t$ for $x \in \Omega$. Consider the prescribed mean curvature Dirichlet problem of finding a function $f \in C^{2}(\Omega) \cap C^{0}(\bar{\Omega})$ which satisfies

$$
\begin{aligned}
\operatorname{div}(T f) & =H(x, f) & & \text { in } \Omega, \\
f & =\phi & & \text { on } \partial \Omega,
\end{aligned}
$$

where $\phi \in C^{0}(\partial \Omega)$ is a prescribed function and

$$
T f=\frac{\nabla f}{\sqrt{1+|\nabla f|^{2}}}
$$

such a function $f$, if it exists, is a classical solution of the Dirichlet problem. It has long been known (e.g., Bernstein in 1912) that some type of boundary curvature condition (which depends on $H$ ) must be satisfied in order to guarantee that a classical solution exists for each $\phi \in C^{0}(\partial \Omega)$ (e.g., [Jenkins and Serrin 1968; Serrin

MSC2010: primary 76B45, 35J93; secondary 35J62, 53A10.

Keywords: prescribed mean curvature, nonconvex corner, Dirichlet problem. 
1969]). When $H \equiv 0$ and $\partial \Omega$ is smooth, this curvature condition is that $\partial \Omega$ must have nonnegative mean curvature (with respect to the interior normal direction of $\Omega$ ) at each point [Jenkins and Serrin 1968]. However, Leon Simon [1976] has shown that if $\Gamma_{0} \subset \partial \Omega$ is smooth (i.e., $C^{4}$ ), the mean curvature $\Lambda$ of $\partial \Omega$ is negative on $\Gamma_{0}$, and $\Gamma$ is a compact subset of $\Gamma_{0}$, then the minimal hypersurface $z=f(x), x \in \Omega$, extends to $\Omega \cup \Gamma$ as a continuous function, even though $f$ may not equal $\phi$ on $\Gamma$. Since [Simon 1976] appeared, the requirement that $H \equiv 0$ has been eliminated and the conclusion remains similar to that which Simon reached (see, for example, [Bourni 2011; Lau and Lin 1985; Lin 1987]).

How important is the role of boundary smoothness in the conclusions reached in [Simon 1976]? We shall show, by constructing suitable domains $\Omega$ and Dirichlet data $\phi$, that the existence of a "nonconvex corner" $P$ in $\Gamma$ can cause the unique generalized (e.g., variational) solution to be discontinuous at $P$ even if $\Gamma \backslash\{P\}$ is smooth and the generalized mean curvature $\Lambda^{*}$ (i.e., [Serrin 1969]) of $\Gamma$ at $P$ is $-\infty$; this shows that some degree of smoothness of $\Gamma$ is required to obtain the conclusions in [Simon 1976]. We shall prove the following.

Theorem 1. Let $n \in \mathbb{N}, n \geq 2$, and assume there exists $\lambda>0$ such that $|H(x, t)| \leq \lambda$ for $x \in \mathbb{R}^{n}$ and $t \in \mathbb{R}$. Then there exist a domain $\Omega \subset \mathbb{R}^{n}$ and a point $P \in \partial \Omega$ such that

(i) $\partial \Omega \backslash\{P\}$ is smooth $\left(C^{\infty}\right)$,

(ii) there is a neighborhood $\mathcal{N}$ of $P$ such that $\Lambda(x)<0$ for $x \in \mathcal{N} \cap \partial \Omega \backslash\{P\}$, where $\Lambda$ is the mean curvature of $\partial \Omega$, and

(iii) $\Lambda^{*}(P)=-\infty$, where $\Lambda^{*}$ is the generalized mean curvature of $\partial \Omega$,

and there exists Dirichlet boundary data $\phi \in C^{\infty}\left(\mathbb{R}^{n}\right)$ such that the minimizer $f \in \mathrm{BV}(\Omega)$ of

$$
J(u)=\int_{\Omega}|D u|+\int_{\Omega} \int_{0}^{u} H(x, t) d t d x+\int_{\partial \Omega}|u-\phi| d \mathscr{H}^{n-1}, \quad u \in \mathrm{BV}(\Omega),
$$

exists and satisfies $(1), f \in C^{2}(\Omega) \cap C^{0}(\bar{\Omega} \backslash\{P\}) \cap L^{\infty}(\Omega), f \notin C^{0}(\bar{\Omega})$, and $f \neq \phi$ in a neighborhood of $P$ in $\partial \Omega$.

Since there are certainly many examples of Dirichlet problems which have continuous solutions even though their domains fail to satisfy appropriate smoothness or boundary curvature conditions (e.g., by restricting to a smaller domain a classical solution of a Dirichlet problem on a larger domain), the question of necessary or sufficient conditions for the continuity at $P$ of a generalized solution of a particular Dirichlet problem is of interest and the examples here suggest (to us) that a "ConcusFinn" type condition might yield necessary conditions for the continuity at $P$ of solutions (see Section 5). 
We view this note as analogous to other articles (e.g., [Shi and Finn 2004; Huff and McCuan 2006; 2009; Korevaar 1980]) which enhance our knowledge of the behavior of solutions of boundary value problems for prescribed mean curvature equations by constructing and analyzing specific examples. One might also compare Theorem 1 with the behavior of generalized solutions of (1)-(2) when $\partial \Omega \backslash\{P\}$ is smooth and $|H(x, \phi(x))| \leq(n-1) \Lambda(x)$ for $x \in \partial \Omega \backslash\{P\}$ (e.g., [Elcrat and Lancaster 1986; Lancaster $1985 ; 1988]$ ) and with capillary surfaces (e.g., [Lancaster and Siegel 1996]).

\section{Nonparametric minimal surfaces in $\mathbb{R}^{3}$}

In this section, we will assume $n=2$ and $H \equiv 0$; this allows us to use explicit comparison functions and illustrate our general procedure. Let $\Omega$ be a bounded, open set in $\mathbb{R}^{2}$ with locally Lipschitz boundary $\partial \Omega$ such that a point $P$ lies on $\partial \Omega$ and there exist distinct rays $l^{ \pm}$starting at $P$ such that $\partial \Omega$ is tangent to $l^{+} \cup l^{-}$at $P$. By rotating and translating the domain, we may assume $P=(0,1)$ and there exists a $\sigma \in\left(-\frac{\pi}{2}, \frac{\pi}{2}\right)$ such that

$$
\begin{aligned}
& l^{-}=\{(r \cos (\sigma), 1+r \sin (\sigma)): r \geq 0\}, \\
& l^{+}=\{(r \cos (\pi-\sigma), 1+r \sin (\pi-\sigma)): r \geq 0\},
\end{aligned}
$$

$$
\Omega \cap B(P, \delta)=\left\{(r \cos (\theta), 1+r \sin (\theta)): 0<r<\delta, \theta^{-}(r)<\theta<\theta^{+}(r)\right\}
$$

for some $\delta>0$ and functions $\theta^{ \pm} \in C^{0}([0, \delta))$ which satisfy $\theta^{-}<\theta^{+}, \theta^{-}(0)=\sigma$ and $\theta^{+}(0)=\pi-\sigma$; here $B(P, \delta)$ is the open ball in $\mathbb{R}^{2}$ centered at $P$ of radius $\delta$. If we set $\alpha=\frac{\pi}{2}-\sigma$, then $\alpha \in(0, \pi)$ and the angle at $P$ in $\Omega$ of $\partial \Omega$ has size $2 \alpha$. As $\sigma<0$ goes to zero, $2 \alpha>\pi$ goes to $\pi$ and the (upper) region between $l^{-}$and $l^{+}$ becomes "less nonconvex" and approaches a half-plane through $P$. We will show that for each choice of $\sigma \in\left(-\frac{\pi}{2}, 0\right)$, there is a domain $\Omega$ as above and a choice of Dirichlet data $\phi \in C^{\infty}(\partial \Omega)$ such that the solution of (1)-(2) for $\Omega$ and $\phi$ is discontinuous at $P$.

Fix $\sigma \in\left(-\frac{\pi}{2},-\frac{\pi}{4}\right)$. Let $\epsilon$ be a small, fixed parameter, say $\epsilon \in(0,0.5)$, and let $a=a(\sigma) \in(1,2)$ be a parameter to be determined. Set $\tau=(1+\epsilon) \cot (-\sigma)$ and $r_{1}=\sqrt{\tau^{2}+(1+\epsilon)^{2}}$. Define $h_{2 / \pi} \in C^{2}((0,2) \times(-1,1))$ by

$$
h_{2 / \pi}\left(x_{1}, x_{2}\right)=\frac{2}{\pi} \ln \left(\frac{\cos \left(\frac{\pi x_{2}}{2}\right)}{\sin \left(\frac{\pi x_{1}}{2}\right)}\right) \text {. }
$$

Notice that the graph of $h_{2 / \pi}$ is part of Scherk's first surface, so $\operatorname{div}\left(T h_{2 / \pi}\right)=0$ on $(0,2) \times(-1,1)$, and $h_{2 / \pi}(t, t-1)=0$ for each $t \in(0,2)$. A computation using L'Hospital's Rule shows

$$
\lim _{t \rightarrow 0^{+}} h_{2 / \pi}((t \cos (\theta), 1+t \sin (\theta)))=\frac{2}{\pi} \ln (-\tan (\theta)), \quad \theta \in\left(-\frac{\pi}{2}, 0\right) .
$$




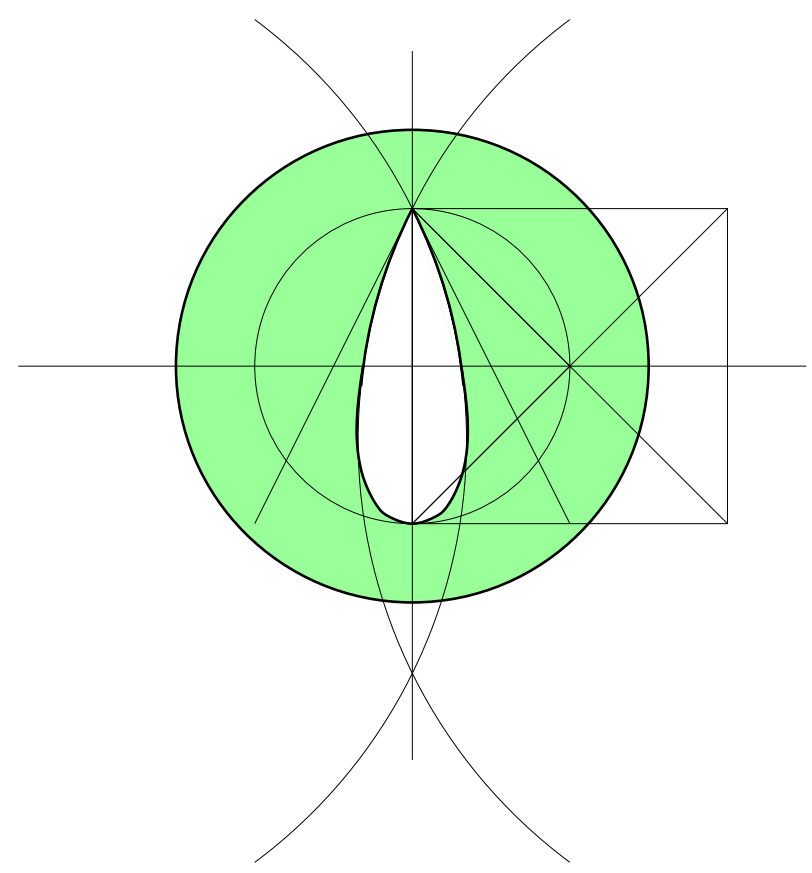

Figure 1. $\Omega$.

Let $D=B(\mathcal{O}, 1) \cap B\left((\tau,-\epsilon), r_{1}\right) \cap B\left((-\tau,-\epsilon), r_{1}\right)$ be the intersection of three open disks and let $E \subset D$ be a strictly convex domain such that $\left\{x \in \partial E: x_{2}<1\right\}$ is a $C^{\infty}$ curve, $E \cap\left\{x_{2} \geq 0\right\}=D \cap\left\{x_{2} \geq 0\right\}, E$ is symmetric with respect to the $x_{2}$-axis, and $(0,-1) \in \partial E$; here 0 denotes $(0,0)$. Define

$$
\Omega=B(\mathcal{O}, a) \backslash \bar{E}
$$

(see Figure 1); notice that $P \in \partial \Omega$ and (4) holds with the choice of $\sigma$ above. If we set $C=\left\{\left(x_{1}, x_{2}\right) \in \mathbb{R}^{2}: 0<x_{1}<1, x_{1}-1<x_{2}<1-x_{1}\right\}$, then (5) implies $\sup _{x \in C \cap \partial E} h_{2 / \pi}(x)<\infty$.

Let

$$
m>\max \left\{r_{1} \cosh ^{-1}\left(\frac{2+\sqrt{\tau^{2}+\epsilon^{2}}}{r_{1}}\right), \sup _{x \in C \cap \partial E} h_{2 / \pi}(x)\right\} .
$$

Notice that $m$ is independent of the parameter $a$. Define $\phi \in C^{\infty}(\partial \Omega)$ by $\phi=0$ on $\partial B(0, a)$ and $\phi=m$ on $\partial E$. Let $f$ be the variational solution of (1)-(2) with $\phi$ as given here (e.g., [Gerhardt 1974; Giusti 1978]). Since $\phi \geq 0$ on $\partial \Omega$ and $\phi>0$ on $\partial E, f \geq 0$ in $\Omega$ (e.g., Lemma 2 (with $h \equiv 0$ )) and so $f>0$ in $\Omega$ (e.g., the Hopf boundary point lemma). Notice that $h_{2 / \pi}=0<f$ on $\Omega \cap \partial C$ and $h_{2 / \pi}<\phi$ on $C \cap \partial E=C \cap \partial \Omega$, and therefore $h_{2 / \pi}<f$ on $\Omega \cap C$ (see Figure 2). Together with 


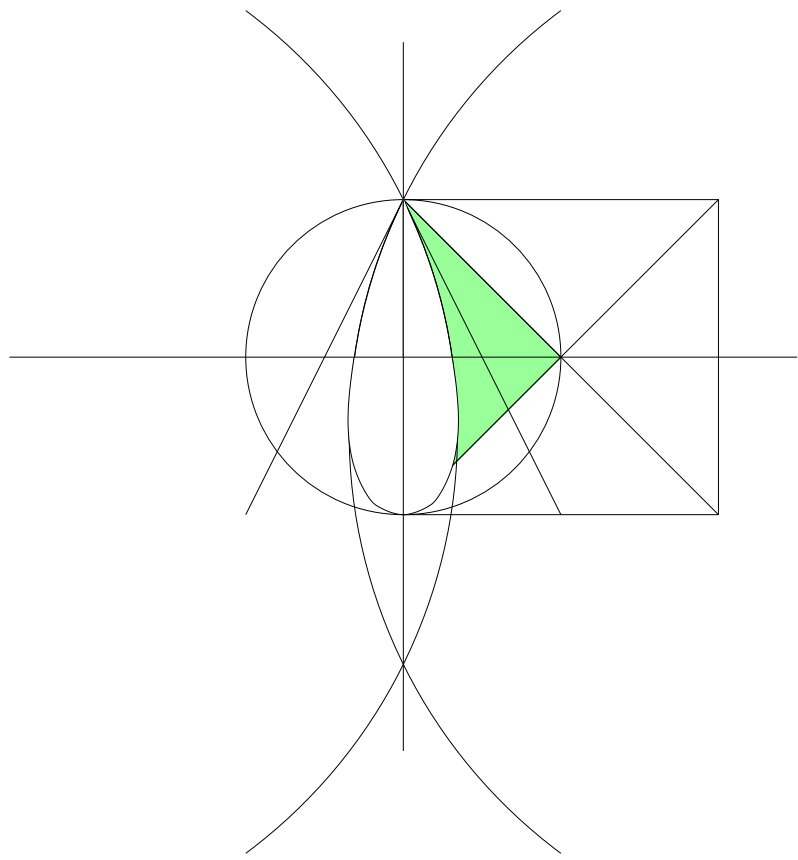

Figure 2. $\Omega \cap C$, the domain of the comparison function for (6).

(5), this implies

$$
\liminf _{\Omega \cap C \ni x \rightarrow P} f(x) \geq \frac{2}{\pi} \ln (\tan (-\sigma))>0 .
$$

Set $W=B(\mathbb{O}, a) \backslash \overline{B(0,1)}$ (see Figure 3); then $W \subset \Omega$. Define the function $g \in C^{\infty}(W) \cap C^{0}(\bar{W})$ by $g(x)=\cosh ^{-1}(a)-\cosh ^{-1}(|x|)$ and notice that the graph of $g$ is part of a catenoid, where $g=0$ on $\partial B(\mathbb{O}, a)$ and $g=\cosh ^{-1}(a)$ on $\partial B(\mathbb{O}, 1)$. It follows from the general comparison principle (e.g., [Finn 1986, Theorem 5.1]) that $f \leq g$ on $W$ and therefore

$$
f \leq \cosh ^{-1}(a) \text { on } W .
$$

If we select $a>1$ so that $\cosh ^{-1}(a)<\frac{2}{\pi} \ln (\tan (-\sigma))$, then (6) and (7) imply that $f$ cannot be continuous at $P$. Notice that [Simon 1976] implies $f \in C^{0}(\bar{\Omega} \backslash\{P\})$.

This example illustrates the procedure we shall use in Section 4; a somewhat similar approach was used in [Shi and Finn 2004; Korevaar 1980; Lancaster and Siegel 1996; Serrin 1969]. The case $\sigma \in\left[-\frac{\pi}{4}, 0\right)$ has a similar proof with the changes that $D$ is the intersection of the open disk $B(0,1)$ with the interiors of two ellipses, and a Scherk surface with rhomboidal domain [Nitsche 1989, pp. 70-71] is used as a comparison surface to obtain the analog of (6); the details can be found in [Melin 2013]. 


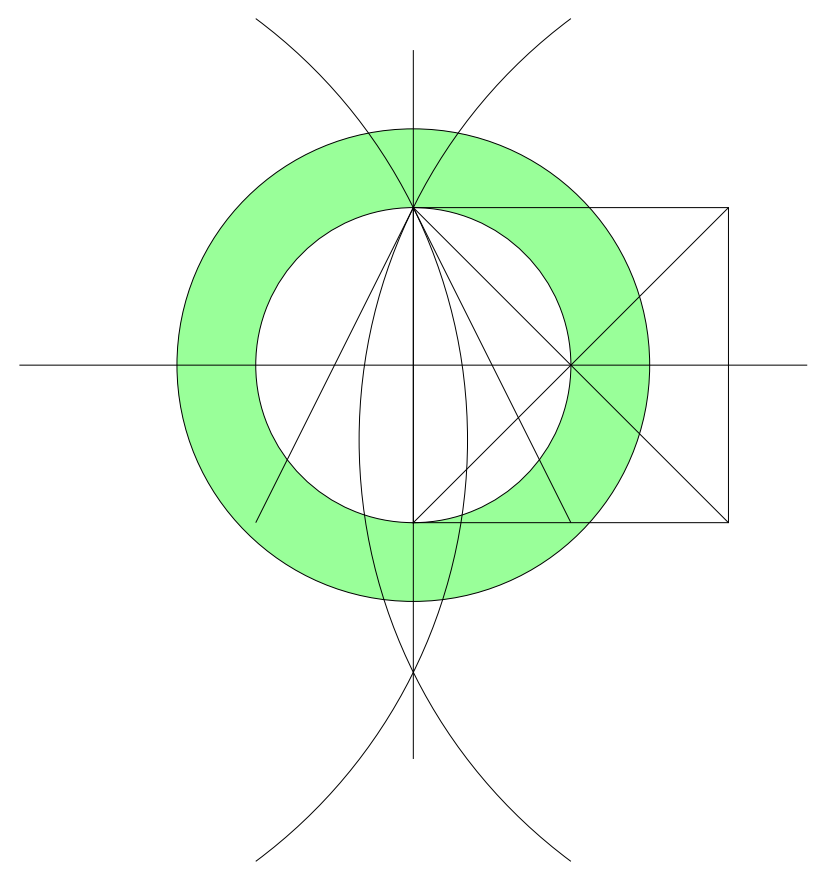

Figure 3. $W$, the domain of the comparison function for (7).

\section{Lemmata}

Lemma 1. Let $\Omega$ be a bounded open set in $\mathbb{R}^{n}, n \geq 2$, with locally Lipschitz boundary and let $\Gamma$ be an open $C^{2}$ subset of $\partial \Omega$. Let $\phi \in L^{\infty}(\partial \Omega) \cap C^{1, \beta}(\Gamma)$. Suppose $g \in C^{2}(\Omega) \cap L^{\infty}(\Omega)$ is the variational solution of (1)-(2) and $g<\phi$ on $\Gamma$. Then

$$
v \equiv \frac{(\nabla g,-1)}{\sqrt{1+|\nabla g|^{2}}} \in C^{0}(\Omega \cup \Gamma)
$$

and $v \cdot \eta=1$ on $\Gamma$, where $\eta(x) \in S^{n-1}$ is the exterior unit normal to $\Gamma$ at $x$.

Proof. Since $g$ minimizes the functional $J$ in (3) over BV $(\Omega), g$ also minimizes the functional $K(u)=J(u)-\int_{\Gamma} \phi d \mathscr{H}^{n-1}$. Notice

$$
K(u)=\int_{\Omega}|D u|+\int_{\Omega} \int_{0}^{u} H(x, t) d t d x+\int_{\partial \Omega \backslash \Gamma}|u-\phi| d \mathscr{H}^{n-1}-\int_{\Gamma} u d \mathscr{H}^{n-1}
$$

for each $u \in \mathrm{BV}(\Omega)$ with $\operatorname{tr}(u) \leq \phi$ on $\Gamma$; in particular, this holds when $u=g$. Therefore, for each $x \in \Gamma$, there exists $\rho>0$ such that $\partial \Omega \cap B_{n}(x, \rho) \subset \Gamma$, and the lemma follows as in [Korevaar and Simon 1996].

Lemma 2. Let $\Omega$ be a bounded open set in $\mathbb{R}^{n}, n \geq 2$, with locally Lipschitz boundary, $\phi, \psi \in L^{\infty}(\partial \Omega)$ with $\psi \leq \phi$ on $\partial \Omega, H_{0} \in C^{2}(\Omega \times \mathbb{R})$ with $H_{0}(x, t)$ 
nondecreasing in $t$ for $x \in \Omega$, and $H_{0} \geq H$ on $\Omega \times \mathbb{R}$. Consider the boundary value problem

$$
\begin{aligned}
\operatorname{div}(T f) & =H_{0}(x, f) & & \text { in } \Omega, \\
f & =\psi & & \text { on } \partial \Omega .
\end{aligned}
$$

Suppose $g \in C^{2}(\Omega) \cap L^{\infty}(\Omega)$ is the variational solution of (1)-(2) and either

(i) $h \in C^{2}(\Omega) \cap L^{\infty}(\Omega)$ is the variational solution of (8)-(9), or

(ii) $\psi \in C^{0}(\partial \Omega), h \in C^{2}(\Omega) \cap C^{0}(\bar{\Omega})$, and $h$ satisfies (8)-(9).

Then $h \leq g$ in $\Omega$.

Proof. Let $A=\{x \in \Omega: h(x)>g(x)\}$. In case (i), let $f=h I_{\Omega \backslash A}+g I_{A}$, where $I_{B}$ is the characteristic function of a set $B$; then a simple calculation using $J(g) \leq J(f)$ shows that $J_{1}(f) \leq J_{1}(h)$ and therefore $f=h$ and $A=\varnothing$, where

$$
J_{1}(u)=\int_{\Omega}|D u|+\int_{\Omega} \int_{0}^{u} H_{0}(x, t) d t d x+\int_{\partial \Omega}|u-\psi| d \mathscr{H}^{n-1}, \quad u \in \mathrm{BV}(\Omega),
$$

is the functional which $h$ minimizes. In case (ii), the conclusion follows from Lemma 1 of [Williams 1978].

Lemma 3. Let $\Omega \subset\left\{x \in \mathbb{R}^{2}: x_{2}>0\right\}$ be a bounded open set, $n \in \mathbb{N}$ with $n \geq 2$, and $g \in C^{2}(\Omega)$. Set $\widetilde{\Omega}=\left\{\left(x_{1}, x_{2} \omega\right) \in \mathbb{R}^{n}:\left(x_{1}, x_{2}\right) \in \Omega, \omega \in S^{n-2}\right\}$ and define $\tilde{g} \in C^{2}(\widetilde{\Omega})$ by $\tilde{g}\left(x_{1}, x_{2} \omega\right)=g\left(x_{1}, x_{2}\right)$ for $\left(x_{1}, x_{2}\right) \in \Omega, \omega \in S^{n-2}$. Then, for

$$
x=\left(x_{1}, \ldots, x_{n}\right)=\left(x_{1}, r \omega\right) \in \widetilde{\Omega}
$$

with $r=\sqrt{x_{2}^{2}+\cdots+x_{n}^{2}}, \omega=\frac{1}{r}\left(x_{2}, \ldots, x_{n}\right)$, and $\left(x_{1}, r\right) \in \Omega$, we have

$$
\operatorname{div}\left(\frac{\nabla \tilde{g}}{\sqrt{1+|\nabla \tilde{g}|^{2}}}\right)(x)=\operatorname{div}\left(\frac{\nabla g}{\sqrt{1+|\nabla g|^{2}}}\right)\left(x_{1}, r\right)+\frac{n-2}{r} \frac{g_{x_{2}}\left(x_{1}, r\right)}{\sqrt{1+\left|\nabla g\left(x_{1}, r\right)\right|^{2}}} .
$$

In particular, if $H \geq 0, R>0, \Omega \subset\left\{x \in \mathbb{R}^{2}: x_{2} \geq R\right\}$, and

$$
\operatorname{div}\left(\frac{\nabla g}{\sqrt{1+|\nabla g|^{2}}}\right) \geq H+\frac{n-2}{R} \text { on } \Omega,
$$

then

$$
\operatorname{div}\left(\frac{\nabla \tilde{g}}{\sqrt{1+|\nabla \tilde{g}|^{2}}}\right) \geq H \quad \text { on } \widetilde{\Omega}
$$


Proof. Notice that

$$
\begin{aligned}
1+|\nabla \tilde{g}|^{2} & =1+|\nabla g|^{2}, \\
\left(1+|\nabla \tilde{g}|^{2}\right) \triangle \tilde{g} & =\left(1+|\nabla g|^{2}\right)\left(\triangle g+\frac{n-2}{r} g_{x_{2}}\right), \\
\sum_{i, j=1}^{n} \frac{\partial \tilde{g}}{\partial x_{i}} \frac{\partial \tilde{g}}{\partial x_{j}} \frac{\partial^{2} \tilde{g}}{\partial x_{i} \partial x_{j}} & =\left(\frac{\partial g}{\partial x_{1}}\right)^{2} \frac{\partial^{2} g}{\partial x_{1}^{2}}+2 \frac{\partial g}{\partial x_{1}} \frac{\partial g}{\partial x_{2}} \frac{\partial^{2} g}{\partial x_{1} \partial x_{2}}+\left(\frac{\partial g}{\partial x_{2}}\right)^{2} \frac{\partial^{2} g}{\partial x_{2}^{2}},
\end{aligned}
$$

and so

$$
\begin{aligned}
& \left(1+|\nabla \tilde{g}|^{2}\right) \triangle \tilde{g}-\sum_{i, j=1}^{n} \frac{\partial \tilde{g}}{\partial x_{i}} \frac{\partial \tilde{g}}{\partial x_{j}} \frac{\partial^{2} \tilde{g}}{\partial x_{i} \partial x_{j}} \\
& \quad=\left(1+g_{x_{2}}^{2}\right) g_{x_{1} x_{1}}-2 g_{x_{1}} g_{x_{2}} g_{x_{1} x_{2}}+\left(1+g_{x_{1}}^{2}\right) g_{x_{2} x_{2}}+\frac{n-2}{r}\left(1+g_{x_{1}}^{2}+g_{x_{2}}^{2}\right) g_{x_{2}} .
\end{aligned}
$$

The lemma follows from this.

\section{The $n$-dimensional case}

Let $B_{k}(x, r)$ denote the open ball in $\mathbb{R}^{k}$ centered at $x \in \mathbb{R}^{k}$ with radius $r>0$ and $\mathrm{O}_{k}=(0, \ldots, 0) \in \mathbb{R}^{k}$, for $k \in \mathbb{N}$. Now consider $n \geq 2$ and set

$$
\lambda=\sup _{(x, t) \in \mathbb{R}^{n} \times \mathbb{R}}|H(x, t)| ;
$$

if $\lambda=0$, replace it with a positive constant. For each $a \in\left(0, \frac{n}{\lambda}\right)$ and $Q \in \mathbb{R}^{n}$, we have

$$
\int_{B_{n}(Q, a)} \lambda^{n} d x<n^{n} \omega_{n}
$$

By translating our problem in $\mathbb{R}^{n}$, we may (and will) assume $Q=0_{n}$. By Proposition 1.1 and Theorem 2.1 of [Giusti 1976], we see that if $\Omega$ is a bounded, connected, and open set in $\mathbb{R}^{n}$ with Lipschitz-continuous boundary, $\bar{\Omega} \subset B_{n}\left(\mathcal{O}_{n}, \frac{n}{\lambda}\right)$, and $\phi \in L^{1}(\partial \Omega)$, then the functional $J$ in (3) has a minimizer $f \in \operatorname{BV}(\Omega), f \in C^{2}(\Omega)$ satisfying (1).

The proof in Section 4.1 consists of setting some parameters (e.g., $p, r_{1}, r_{2}, m_{0}$, $b, c, \tau, \sigma, a)$, determining the domain $\Omega$, finding different comparison functions (e.g., $g_{1}, g^{[u]}, k_{ \pm}, k_{2}, k_{3}, k_{4}$ ), and mimicking (6) and (7) to show that the variational solution $f$ of (1)-(2) is discontinuous at a nonconvex corner. In particular, we use a torus (i.e., $j_{a}$ ) to obtain (21), unduloids (i.e., $k_{ \pm}, k_{2}$ ) to obtain (24) (an analog of (7)), and nodoids (i.e., $g_{1}, g^{[u]}$ ), unduloids (i.e., $k_{ \pm}, k_{4}$ ), and a helicoidal function (i.e., $h_{2}$ ) to obtain (30) (an analog of (6)) and prove that $f$ is discontinuous at $P=(0, p, 0, \ldots, 0) \in \mathbb{R}^{n} \in \partial \Omega$. 
4.1. Codimension 1 singular set. In this section, we will obtain a domain $\Omega$ as above and $\phi \in C^{\infty}\left(\mathbb{R}^{n}\right)$ such that $P \in \partial \Omega$, the minimizer $f$ of (3) is discontinuous at $P, \partial \Omega \backslash T$ is smooth $\left(C^{\infty}\right)$, and $f \in C^{2}(\Omega) \cap C^{0}(\bar{\Omega} \backslash T)$, where $T$ is a smooth set of dimension $n-2$ (i.e., $T$ has codimension 1 in $\partial \Omega$ ). We will use portions of nodoids, unduloids, and helicoidal surfaces with constant mean curvature as comparison functions. For the convenience of the reader, we will denote functions whose graphs are subsets of nodoids with the letter $g$ (e.g., $\left.g_{1}\left(x_{1}, x_{2}\right)\right)$, subsets of CMC helicoids with the letter $h$, and subsets of unduloids (or onduloids) with the letter $k$.

Let $\mathcal{N}_{1} \subset \mathbb{R}^{3}$ be a nodoid which is symmetric with respect to the $x_{3}$-axis and has mean curvature 1 (when $\mathcal{N}_{1}$ is oriented "inward", so that the unit normal $\vec{N}_{\mathcal{N}_{1}}$ to $\mathcal{N}_{1}$ points toward the $x_{3}$-axis at the points of $\mathcal{N}_{1}$ which are furthest from the $x_{3}$-axis). Let $s_{1}=\inf _{(x, t) \in \mathcal{N}_{1}}|x|$ be the inner neck size of $\mathcal{N}_{1}$ and let $s_{3}$ satisfy the condition that the unit normal to $\mathcal{N}_{1}$ is vertical (i.e., parallel to the $x_{3}$-axis) at each point $(x, t) \in \mathbb{R}^{2} \times \mathbb{R}$ of $\mathcal{N}_{1}$ at which $|x|=s_{3}$; then $s_{1}<s_{3}$. Let $s_{2} \in\left(s_{1}, s_{3}\right)$. (Notice that we can assume $s_{2} / s_{1}$ is close to $s_{3} / s_{1}$ if we wish.)

Let us fix $0<p<\frac{1}{\lambda}$ and set $w=(0, p) \in \mathbb{R}^{2}, P=(0, p, 0, \ldots, 0) \in \mathbb{R}^{n}$. Let $m_{0}=\lambda / 2+(n-2) /(p / 3)$. We shall assume $r_{2}=s_{2} / m_{0}<p / 3$; if necessary, we may increase $m_{0}$ to accomplish this. Let $r_{1}=s_{1} / m_{0}$ and $r_{3}=s_{3} / m_{0}$. Let $\mathcal{N}=\left\{\left(m_{0}\right)^{-1} X \in \mathbb{R}^{3}: X \in \mathcal{N}_{1}\right\}$; then $\mathcal{N}$ is a nodoid with mean curvature $m_{0}$. Set $\Delta_{1}=\left\{x \in \mathbb{R}^{2}: r_{1}<|x|<r_{2}\right\}$. Fix $b \in\left(0, \frac{1}{4 m_{0}}\left(1+2 m_{0} p-\sqrt{1+4 m_{0}^{2} p^{2}}\right)\right)$.

Define $g_{1} \in C^{\infty}\left(\Delta_{1}\right) \cap C^{0}\left(\overline{\Delta_{1}}\right)$ to be a function whose graph is a subset of $\mathcal{N}$ on which $\vec{N}_{\mathcal{N}}=\left(n_{1}, n_{2}, n_{3}\right)$ satisfies $n_{3} \geq 0$; then

$$
\operatorname{div}\left(\frac{\nabla g_{1}}{\sqrt{1+\left|\nabla g_{1}\right|^{2}}}\right)=m_{0} \geq \lambda+\frac{2(n-2)}{p / 3} .
$$

By moving $\mathcal{N}$ vertically, we may assume $g_{1}(x)=0$ when $|x|=r_{2}$; then $g_{1}>0$ in $\Delta_{1}$. Notice that $\frac{\partial g_{1}}{\partial x_{1}}\left(r_{1}, 0\right)=-\infty$ and $\frac{\partial g_{1}}{\partial x_{1}}\left(r_{2}, 0\right)<0$; then there exists a $\beta_{0}>0$ such that, for each $\theta \in \mathbb{R}$,

$$
\frac{\partial}{\partial r}\left(g_{1}(r \Theta)\right)<-\beta_{0} \quad \text { for } r_{1}<r<r_{2},
$$

where $\Theta=(\cos (\theta), \sin (\theta))$. Fix $\beta \in\left(0, \beta_{0}\right)$. Let

$$
0<\tau<\min \left\{\frac{p r_{1}}{\sqrt{r_{2}^{2}-r_{1}^{2}}}, \frac{2(1-p \lambda)}{\lambda(2-p \lambda)}, \frac{b(4 p-b)}{4(2 p-b)}\right\} .
$$

Consider $\sigma \in\left(-\frac{\pi}{2}, 0\right)$. Notice that the distance between $L$ and the point $\left(0, p-r_{2}\right)$ is $r_{2} \cos (\sigma)$, where $L$ is the closed sector given by

$$
L=\{(r \cos (\theta), p+r \sin (\theta)): r \geq 0, \sigma \leq \theta \leq \pi-\sigma\} .
$$


Define $r_{4}=\sqrt{p^{2}+\tau^{2}}$ and

$$
M=B_{2}\left((\tau, 0), r_{4}\right) \cap B_{2}\left((-\tau, 0), r_{4}\right) .
$$

Notice that

$$
\tau<\frac{b(4 p-b)}{4(2 p-b)}
$$

and therefore $B_{2}\left(O_{2}, \frac{1}{2}(a+p)-b\right) \subset M$ if $p<a<p+b$.

Set $\sigma=-\arctan (\tau / p)$; then $\cos (\sigma)>r_{1} / r_{2}$, since

$$
\tau<\frac{p \sqrt{r_{2}^{2}-r_{1}^{2}}}{r_{1}}
$$

and $L \cap \overline{B_{2}}=\varnothing$, where $B_{2}=B_{2}\left(\left(0, p-r_{2}\right), r_{1}\right)$. Therefore there exists a $\delta_{1}>0$ such that if $u=\left(u_{1}, u_{2}\right) \in \partial B_{2}\left(O_{2}, p\right)$ with $|u-w|<\delta_{1}$, then

$$
B_{2}\left(\frac{p-r_{2}}{p} u, r_{1}\right) \subset M \text {. }
$$

Since

$$
\tau<\frac{2(1-p \lambda)}{\lambda(2-p \lambda)}
$$

we have $\tau-\left(\frac{2}{\lambda}-r_{4}\right)<-p$ and so $B_{2}\left(\mathscr{O}_{2}, p\right) \subset B_{2}\left((\tau, 0), \frac{2}{\lambda}-r_{4}\right)$ (see Figure 8, right). Notice that

$$
M \backslash\{(0, \pm p)\}=\left\{(r \cos (\theta), p+r \sin (\theta)): 0<r<2 p, \theta^{-}(r)<\theta<\theta^{+}(r)\right\}
$$
for some $\theta^{ \pm} \in C^{0}([0, \delta))$ satisfying $\theta^{-}<\theta^{+}, \theta^{-}(0)=-\pi-\sigma$, and $\theta^{+}(0)=\sigma$.

Let $a>p$ and set $\mathscr{T}=\left\{\left(\left(\frac{1}{2}(a+p)+b \cos v\right) \cos u,\left(\frac{1}{2}(a+p)+b \cos v\right) \sin u, b \sin v+c\right):(u, v) \in R\right\}$, where $R=[0,2 \pi] \times[-\pi, 0]$ and $0<c<b$; since $b<\frac{1}{4 m_{0}}\left(1+2 m_{0} p-\sqrt{1+4 m_{0}^{2} p^{2}}\right)$, we see that

$$
\frac{\frac{1}{2}(a+p)-2 b}{4 b\left(\frac{1}{2}(a+p)-b\right)}>m_{0}
$$

for all $a \geq p$. We shall assume

$$
a \in\left(p, \min \left\{p+b, \frac{1}{\lambda}\right\}\right)
$$

and $c=\sqrt{b^{2}-\left(\frac{1}{2}(a-p)\right)^{2}}$. Notice that $\mathcal{T}$ is the lower half of a torus whose mean curvature (i.e., one half of the trace of the shape operator) at each point is greater than $m_{0}$. Let $\mathscr{T}$ be the graph of a function $j_{a}$ over

$$
\Delta_{a}=\left\{x \in \mathbb{R}^{2}: \frac{1}{2}(a+p)-b \leq|x| \leq \frac{1}{2}(a+p)+b\right\}
$$




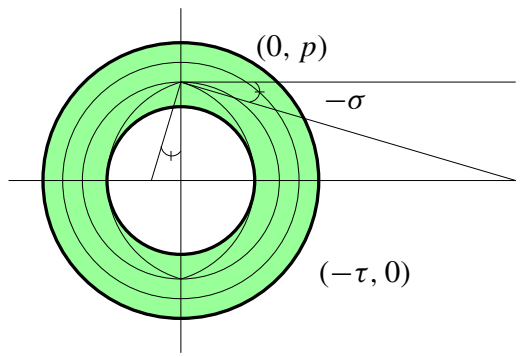

Figure 4. The domain of $j_{a}$.
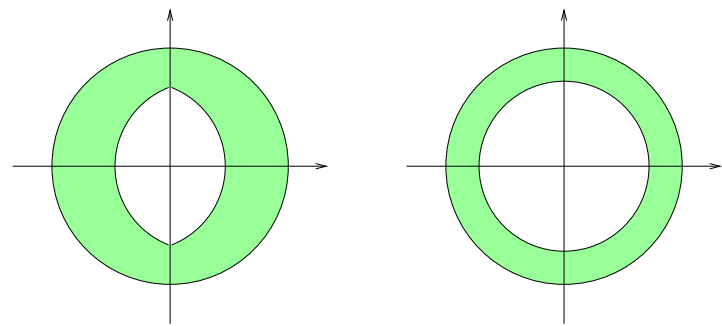

Figure 5. Left: $\Pi_{1, j}(\Omega)$ for $2 \leq j \leq n$. Right: $\Pi_{i, j}(\Omega)$ for $2 \leq i<j \leq n$.

then $j_{a}(x)=0$ on $|x|=a$ and $|x|=p, j_{a}(x)<0$ on $p<|x|<a$, and $j_{a}(x)>0$ on $\frac{1}{2}(a+p)-b \leq|x|<p$ and $a<|x| \leq \frac{1}{2}(a+p)+b$ for $x \in \mathbb{R}^{2}$. Notice that $\left|j_{a}(x)\right|<\frac{1}{2 m_{0}}$ for all $x \in \Delta_{a}$.

Set

$$
\Omega=B_{n}\left(\mathscr{O}_{n}, a\right) \backslash \bar{M},
$$

where $\mathcal{M}=\tilde{M}=\left\{\left(x_{1}, x_{2} \omega\right) \in \mathbb{R}^{n}:\left(x_{1}, x_{2}\right) \in M, \omega \in S^{n-2}\right\}$. If we define

$$
\Pi_{i, j}(A)=\left\{\left(x_{i}, x_{j}\right):\left(x_{1}, \ldots, x_{n}\right) \in A, x_{k}=0 \text { for } k \neq i, j\right\}
$$

for $A \subset \mathbb{R}^{n}$ and $1 \leq i<j \leq n$, then $\Pi_{1, j}(\Omega)=B_{2}\left(\mathcal{O}_{2}, a\right) \backslash \bar{M}$ for $2 \leq j \leq n$ and $\Pi_{i, j}(\Omega)=B_{2}\left(\mathrm{O}_{2}, a\right) \backslash \overline{B_{2}\left(\mathrm{O}_{2}, 1\right)}$ for $2 \leq i<j \leq n$ (see Figure 5).

We wish to select a helicoidal surface in $\mathbb{R}^{3}$ (e.g., [do Carmo and Dajczer 1982]) with constant mean curvature $m_{0}$, axis $\{w\} \times \mathbb{R}$, and pitch $-\beta$ (recall $-\beta \in\left(-\beta_{0}, 0\right)$ ), which we will denote $\mathscr{Y}$; then, for each $t \in \mathbb{R}, k_{t}(\mathscr{Y})=\mathscr{Y}$, where $k_{t}: \mathbb{R}^{3} \rightarrow \mathbb{R}^{3}$ is the helicoidal motion given by $k_{t}\left(x_{1}, x_{2}, x_{3}\right)=\left(l_{t}\left(x_{1}, x_{2}\right), x_{3}-\beta t\right)$ with $l_{t}: \mathbb{R}^{2} \rightarrow \mathbb{R}^{2}$ given by

$$
l_{t}\left(x_{1}, x_{2}\right)=\left(x_{1} \cos (t)+\left(x_{2}-p\right) \sin (t), p-x_{1} \sin (t)+\left(x_{2}-p\right) \cos (t)\right) .
$$

Set $c_{0}=\frac{1}{4} \beta \sigma<0$. By vertically translating $\mathscr{Y}$, we may assume that there is an open $c_{0}$-level curve $\mathscr{L}_{0}$ of $\mathscr{Y}$ with endpoints $w=(0, p)$ and $b=\left(b_{1}, b_{2}\right)$ such that 


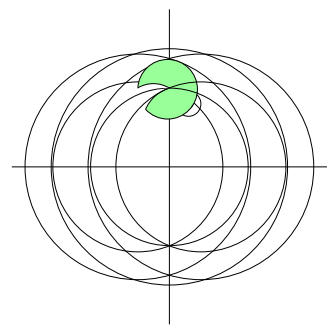

Figure 6. $\mathscr{R}$.

$\mathscr{L}_{0} \subset(0, \infty) \times \mathbb{R}, \mathscr{L}=\overline{\mathscr{L}}_{0}$ is tangent to the (horizontal) line $\mathbb{R} \times\{p\}$ at $w$, and the slope $m_{v}$ of the tangent line to $\mathscr{L}$ at $v$ satisfies $\left|m_{v}\right|<\tan (-\sigma / 5)$ for each $v \in \mathscr{L}_{0}$; then $\mathscr{L} \times\left\{c_{0}\right\} \subset \mathscr{Y}$ and the curves $l_{t}\left(\mathscr{L}_{0}\right),-\frac{7 \pi}{8}<t<\frac{7 \pi}{8}$, are mutually disjoint. Notice that the set

$$
\mathscr{R}=\left\{l_{t}\left(\mathscr{L}_{0}\right):-\frac{7 \pi}{8}<t<\frac{7 \pi}{8}\right\}=\bigcup_{-\frac{7 \pi}{8}<t<\frac{7 \pi}{8}} l_{t}\left(\mathscr{L}_{0}\right)
$$

is an open subset of $\mathbb{R}^{2} \backslash((-\infty, 0] \times\{p\})$ (see Figure 6), $w \in \overline{\mathscr{R}}$, and $\mathscr{S}$ implicitly defines the smooth function $h_{2}$ on $\mathscr{R}$ given by $h_{2}(x)=\frac{\beta}{4}(\sigma-4 t)$ if $x \in l_{t}\left(\mathscr{L}_{0}\right)$ for some $t \in\left(-\frac{\pi}{2}, \frac{\pi}{2}\right)$. Notice that $B_{2}\left(w, b_{1}\right) \cap\left\{x_{1}>0\right\} \subset \mathscr{R}$. Now we have $l_{t}\left(\mathscr{L}_{0}\right) \cap M=\varnothing$ for $t \in\left(\frac{3 \sigma}{4}, \frac{\sigma}{4}\right)$ and, by making $b_{1}>0$ sufficiently small, we may assume that

$$
l_{t}\left(\mathscr{L}_{0}\right) \subset B_{2}\left(\mathscr{O}_{2}, p\right) \backslash M \quad \text { for each } t \in\left(\frac{3 \sigma}{4}, \frac{\sigma}{4}\right) .
$$

Notice that $h_{2}<\beta\left(2 \sigma^{2}-\pi\right) /(8 \sigma)$ on $l_{t}\left(\mathscr{L}_{0}\right)$ for $-\frac{\pi}{2}<t<\frac{7 \pi}{8}$.

Let us fix $u=\left(u_{1}, u_{2}\right) \in \partial B_{2}\left(O_{2}, p\right)$ such that $|u-w|<\min \left\{\delta_{1}, b_{1}\right\}$ and $u_{1}>0$. Then there exists $\theta_{u} \in\left(0, \frac{\pi}{2}\right)$ such that $u=\left(p \cos \left(\theta_{u}\right), p \sin \left(\theta_{u}\right)\right)$. Define

$$
g^{[u]}(x)=g_{1}\left(x+\frac{r_{2}-p}{p} u\right)
$$

and notice that $g^{[u]}(u)=g_{1}\left(\frac{r_{2}}{p} u\right)=0$, since $\left|\frac{r_{2}}{p} u\right|=r_{2}$. Note that the domain

$$
\mathscr{D}^{[u]}=\left\{x+\frac{p-r_{2}}{p} u: x \in \Delta_{1}\right\}=B_{2}\left(\frac{p-r_{2}}{p} u, r_{2}\right) \backslash \overline{B_{2}\left(\frac{p-r_{2}}{p} u, r_{1}\right)}
$$

of $g^{[u]}$ is contained in $B_{2}\left(\mathscr{O}_{2}, p\right)$ since $\partial B_{2}\left(\frac{p-r_{2}}{p} u, r_{2}\right)$ and $\partial B_{2}\left(\mathscr{O}_{2}, p\right)$ are tangent circles at $u$ and $r_{2}<p$ (see Figure 7). Notice that

$$
h_{2}\left(r \cos \left(\theta_{u}\right), r \sin \left(\theta_{u}\right)\right)<g^{[u]}\left(r \cos \left(\theta_{u}\right), r \sin \left(\theta_{u}\right)\right)
$$

when $p-r_{2}+r_{1} \leq r \leq p$, because $h_{2}(u)<0=g^{[u]}(u), \beta<\beta_{0}$, and (12) holds.

Let

$$
\mathcal{N}_{ \pm} \subset\left\{x \in \mathbb{R}^{2}: r_{4} \leq\left|\left(x_{1} \pm \tau, x_{2}\right)\right| \leq \frac{2}{\lambda}-r_{4}\right\} \times \mathbb{R}
$$




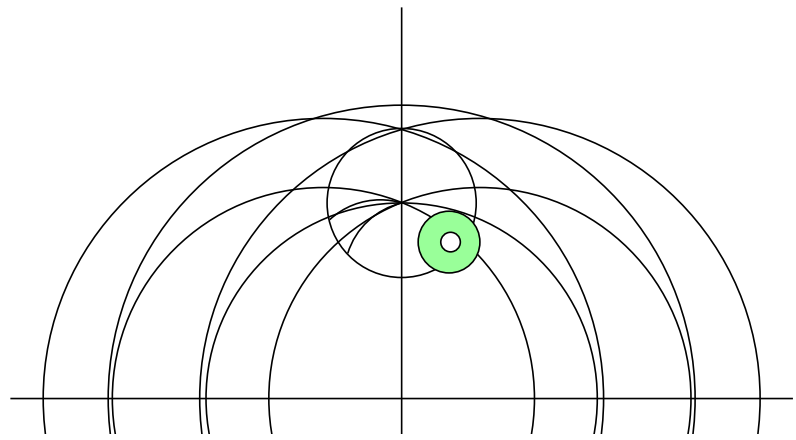

Figure 7. $\mathscr{D}^{[u]} ; \Omega \cap \widetilde{\mathscr{D}}^{[u]}$ is the domain of the comparison function for (28).
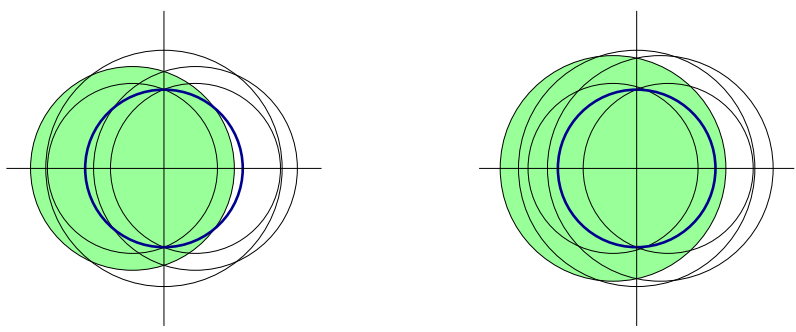

Figure 8. Left: $B_{2}\left(O_{2}, p\right) \nsubseteq B_{2}\left((-\tau, 0), \frac{2}{\lambda}-r_{4}\right)$. Right: $B_{2}\left(\mathrm{O}_{2}, p\right) \subset B_{2}\left((-\tau, 0), \frac{2}{\lambda}-r_{4}\right)$.

be unduloids in $\mathbb{R}^{3}$ with mean curvature $\frac{\lambda}{2}$ such that $\{(\mp \tau, 0)\} \times \mathbb{R}$ are the respective axes of symmetry; the minimum and maximum radii (or "neck" and "waist" sizes) of both unduloids are $r_{4}$ and $\frac{2}{\lambda}-r_{4}$, respectively. Set

$$
\Delta_{ \pm}=B_{2}\left((\mp \tau, 0), \frac{2}{\lambda}-r_{4}\right) \backslash \overline{B_{2}\left((\mp \tau, 0), r_{4}\right)}
$$

and define $k_{ \pm} \in C^{\infty}\left(\Delta_{ \pm}\right)$so that the graphs of $k_{ \pm}$are subsets of $\mathcal{N}_{ \pm}$, respectively,

$$
\operatorname{div}\left(T k_{ \pm}\right)=-\lambda \quad \text { in } \Delta_{ \pm}
$$

$\left.\frac{\partial}{\partial r}\left(k_{ \pm}((\mp p, 0)+r \Theta)\right)\right|_{r=r_{4}}=-\infty$ and $\left.\frac{\partial}{\partial r}\left(k_{ \pm}((\mp p, 0)+r \Theta)\right)\right|_{r=2 / \lambda-r_{4}}=-\infty$ for each $\theta \in \mathbb{R}$, where $\Theta=(\cos (\theta), \sin (\theta))$. We may vertically translate $\mathcal{N}_{ \pm}$so that $k_{ \pm}(x)=0$ for $x \in \mathbb{R}^{2}$ with $\left|\left(x_{1} \pm \tau, x_{2}\right)\right|=\frac{2}{\lambda}-r_{4}$. Notice that $k_{+}(0, p)=k_{-}(0, p)=$ $\sup _{\Delta_{+}} k_{+}=\sup _{\Delta_{-}} k_{-}$.

Let $\mathcal{N} \subset\left\{x \in \mathbb{R}^{2}: p \leq|x| \leq \frac{2}{\lambda}-p\right\} \times \mathbb{R}$ be an unduloid with mean curvature $\frac{\lambda}{2}$ such that the $x_{3}$-axis is the axis of symmetry and the minimum and maximum radii (or "neck" and "waist" sizes) are $p$ and $\frac{2}{\lambda}-p$, respectively. Set

$$
\Delta_{2}=B_{2}\left(\mathrm{O}_{2}, \frac{2}{\lambda}-p\right) \backslash \overline{B_{2}\left(\mathbb{O}_{2}, p\right)}
$$




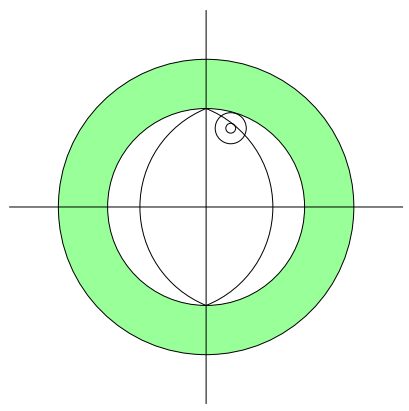

Figure 9. $B_{2}\left(\mathrm{O}_{2}, a\right) \backslash \overline{B_{2}\left(\mathrm{O}_{2}, p\right)}:(22)$.

and define $k_{2} \in C^{\infty}\left(\Delta_{2}\right)$ so that the graph of $k_{2}$ is a subset of $\mathcal{N}, \operatorname{div}\left(T k_{2}\right)=-\lambda$ in $\Delta_{2}$, and $\left.\frac{\partial}{\partial r}\left(k_{2}(r \Theta)\right)\right|_{r=p}=\left.\frac{\partial}{\partial r}\left(k_{2}(r \Theta)\right)\right|_{r=2 / \lambda-p}=-\infty$ for each $\theta \in \mathbb{R}$, where $\Theta=(\cos (\theta), \sin (\theta))$.

Define $\phi \in C^{\infty}\left(\mathbb{R}^{n}\right)$ so that $\phi=0$ on $\partial B_{n}\left(\bigcirc_{n}, a\right)$ and $\phi=m$ on $\partial \mu$, where

$$
m>\max \left\{g_{1}\left(0, r_{1}\right), \frac{1}{2 m_{0}}, k_{+}\left(0, r_{4}-\tau\right)+k_{2}(0, p)-k_{2}\left(0, \frac{2}{\lambda}-p\right)\right\} ;
$$

recall then that $m>j_{a}\left(\frac{1}{2}(a+p)-b\right)$. Let $f$ be the variational solution of (1)-(2) with $\Omega$ and $\phi$ as given here; that is, let $f$ minimize the functional given in (3) and notice that the existence of $f$ follows from (10), (16), §1.D of [Giusti 1976], and [Gerhardt 1974; Giusti 1978]. (Notice that there exists $w: B_{2}\left(0_{2}, a\right) \backslash M \rightarrow \mathbb{R}$ such that $f=\tilde{w}$.) The comparison principle implies $j_{a}(x) \leq f(x)$ for $x \in \Omega$, and so $f(x) \geq j_{a}(x) \geq 0$ if $x \in \Omega$ with $|x| \leq p$ (recall (16) holds). In particular,

$$
f(x) \geq 0 \quad \text { when } x \in \Omega \text { with }|x| \leq p .
$$

Set $W=\left(B_{2}\left(\mathrm{O}_{2}, a\right) \backslash \overline{B_{2}\left(\mathcal{O}_{2}, p\right)}\right) \times \mathbb{R}^{n-2}$. Now

$$
\Omega \subset B_{2}\left(\mathrm{O}_{2}, a\right) \times \mathbb{R}^{n-2} \subset B_{2}\left(\mathrm{O}_{2}, \frac{2}{\lambda}-p\right) \times \mathbb{R}^{n-2}
$$

(see Figure 9). Define $k_{3}(x)=k_{2}\left(x_{1}, x_{2}\right)-k_{2}(0, a)$ for $x=\left(x_{1}, x_{2}, \ldots, x_{n}\right) \in W$. Notice that $f=0 \leq k_{3}$ on $\bar{W} \cap \partial B_{n}\left(\mathbb{O}_{n}, a\right)$,

$$
\operatorname{div}(T f)=H(x, f(x)) \geq-\lambda=\operatorname{div}\left(T k_{3}\right) \quad \text { in } \Omega \cap W,
$$

and $\left.\frac{\partial}{\partial r}\left(k_{2}(r \Theta)\right)\right|_{r=p}=-\infty$ (so that $\lim _{W \ni y \rightarrow x} T k_{3}(y) \cdot \xi(x)=1$, for $\xi$ the unit exterior normal to $\partial W$ and $\left.x \in \partial B_{2}\left(O_{2}, p\right) \times \mathbb{R}^{n-2}\right)$. The general comparison principle (e.g., [Finn 1986, Theorem 5.1]) then implies

$$
f \leq k_{3} \quad \text { in } \Omega \cap W
$$




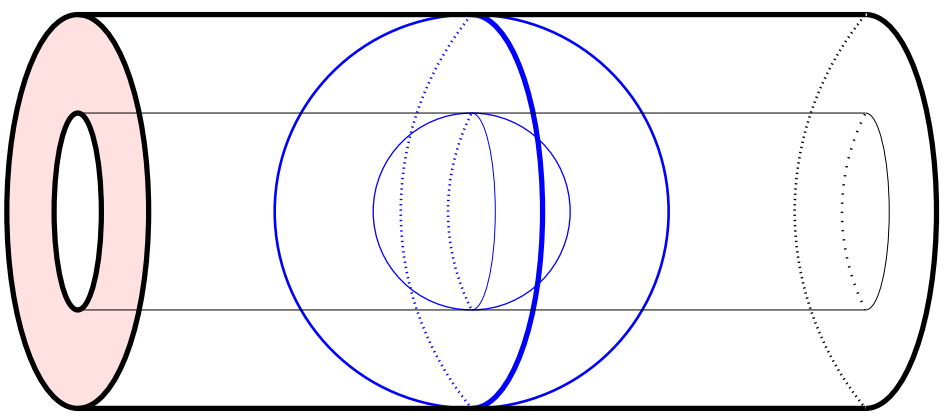

Figure 10. (23): $W$ and $B_{n}\left(\mathscr{O}_{n}, a\right) \backslash \overline{B_{n}\left(\mathscr{O}_{n}, p\right)}$ when $n=3$.

and, in particular,

$$
\limsup _{\Omega \cap W \ni y \rightarrow x} f(y) \leq k_{3}(x) \quad \text { for } x \in \partial \Omega \cap \bar{W}
$$

(see Figure 10). By rotating the axis of symmetry of $W$ through all lines in $\mathbb{R}^{n}$ containing $\mathrm{O}_{n}$ (or, equivalently, keeping $W$ fixed and rotating $\Omega$ about $\mathrm{O}_{n}$ ), we see that

$$
\sup \left\{f(x): x \in B_{n}\left(\mathbb{O}_{n}, a\right) \backslash \overline{B_{n}\left(\mathbb{O}_{n}, p\right)}\right\} \leq k_{2}(0, p)-k_{2}(0, a) .
$$

Now define $k_{4} \in C^{\infty}\left(\Delta_{+} \times \mathbb{R}^{n-2}\right) \cap C^{0}\left(\overline{\Delta_{+}} \times \mathbb{R}^{n-2}\right)$ by

$$
k_{4}(x)=k_{+}\left(x_{1}, x_{2}\right)+k_{2}(0, p)-k_{2}(0, a), \quad x=\left(x_{1}, x_{2}, \ldots, x_{n}\right) \in \overline{\Delta_{+}} \times \mathbb{R}^{n-2} .
$$

Combining (1) and (24) with the facts that $\operatorname{div}\left(T k_{4}\right)=-\lambda$ in $\Delta_{+} \times \mathbb{R}^{n-2}$ and $\lim _{\Delta_{+} \times \mathbb{R}^{n-2} \ni y \rightarrow x} T k_{4}(y) \cdot \xi_{+}(x)=1$ for $x \in \partial B_{2}\left((-\tau, 0), r_{4}\right) \times \mathbb{R}^{n-2}$, where $\xi_{+}$is the inward unit normal to $\partial B_{2}\left((-\tau, 0), r_{4}\right) \times \mathbb{R}^{n-2}$, we see that

$$
f \leq k_{4} \quad \text { in } \Omega \cap\left(\Delta_{+} \times \mathbb{R}^{n-2}\right) .
$$

(If Figure 8 (left) held, then (25) would not be valid.) Now let $L: \mathbb{R}^{n} \rightarrow \mathbb{R}^{n}$ be any rotation about $O_{n}$ which satisfies $L(\Omega)=\Omega$, notice that $f \circ L$ satisfies (1)-(2), and apply the previous argument to obtain $f \circ L \leq k_{4}$ in $\Omega \cap\left(\Delta_{+} \times \mathbb{R}^{n-2}\right)$ and therefore

$$
\sup \{f(x): x \in \partial \mathcal{M}\} \leq k_{4}(p, 0)<m .
$$

From Lemma 1, we see that the downward unit normal $N_{f}$ to the graph of $f$ satisfies $N_{f}=(\nu, 0)$ on $\partial \mathcal{M} \backslash\left\{(0, p \omega): \omega \in S^{n-2}\right\}$ and

$$
\lim _{\Omega \ni y \rightarrow x} T f(y) \cdot v(x)=1 \quad \text { for } x \in \partial \mathcal{M} \backslash\left\{(0, p \omega): \omega \in S^{n-2}\right\} .
$$




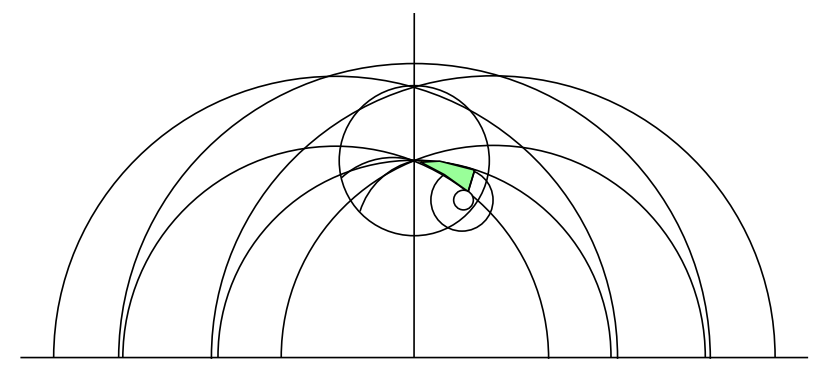

Figure 11. $A$ : (29).

Let us write $B=B_{2}\left(\frac{p-r_{2}}{p} u, r_{2}\right)$; then $\tilde{g}^{[u]}=0 \leq f$ on $\Omega \cap \partial \widetilde{B}$ and $\tilde{g}^{[u]} \leq g_{1}\left(r_{1}, 0\right)<\phi$ on $\widetilde{B} \cap \partial M$. It follows from (1), (11), and Lemma 3 that

$$
\tilde{g}^{[u]}<f \quad \text { on } \Omega \cap \widetilde{D}^{[u]}=\Omega \cap \widetilde{B} .
$$

Set $U=\left\{r(\cos (\theta), \sin (\theta) \omega) \in \Omega: r \in(0, p), \theta \in\left(0, \theta_{u}\right), \omega \in S^{n-2}\right\}$. If we write

$$
\begin{aligned}
& \partial_{1} U=\left\{(p \cos (\theta), p \sin (\theta) \omega): \theta \in\left(0, \theta_{u}\right], \omega \in S^{n-2}\right\}, \\
& \partial_{2} U=\partial M \cap \partial U, \\
& \partial_{3} U=\left\{\left(r \cos \left(\theta_{u}\right), r \sin \left(\theta_{u}\right) \omega\right) \in \bar{\Omega}: r \in[0, p], \omega \in S^{n-2}\right\},
\end{aligned}
$$

then $\partial U=\partial_{1} U \cup \partial_{2} U \cup \partial_{3} U, \tilde{h}_{2} \leq 0 \leq f$ on $\partial_{1} U \backslash\{P\}$, and $\tilde{h}_{2}<\tilde{g}^{[u]}<f$ on $\partial_{3} U$ (see (19)); then (27) and the general comparison principle imply

$$
\tilde{h}_{2}<f \quad \text { in } U=\widetilde{A},
$$

where $A=\left\{r(\cos (\theta), \sin (\theta)) \in B_{2}\left(\mathbb{O}_{2}, p\right) \backslash \bar{M}: r \in(0, p), \theta \in\left(0, \theta_{u}\right)\right\}$ (see Figure 11). Set $\mathscr{R}_{2}=\bigcup_{t=3 \sigma / 4}^{2 \sigma / 4} l_{t}\left(\mathscr{L}_{0}\right)$. Now (18) implies $\widetilde{\mathscr{R}}_{2} \subset U$ and so

$$
f>\tilde{h}_{2} \geq-\frac{\beta \sigma}{4} \quad \text { on } \mathscr{R}_{2}
$$

Using (24) and (30), we see that if $a \in\left(p, \frac{2}{\lambda}-p\right)$ is close enough to $p$, then $k_{2}(0, p)-k_{2}(0, a)<-\frac{\beta \sigma}{4}$ and therefore $f$ cannot be continuous at $P$ or at any point of $T=\left\{(0, p \omega) \in \mathbb{R}^{n}: \omega \in S^{n-2}\right\}$. Note that $f \in C^{0}(\bar{\Omega} \backslash T)$ (e.g., [Lin 1987]).

4.2. One singular point. In this section, we obtain a domain $\Omega$ and $\phi \in C^{\infty}\left(\mathbb{R}^{n}\right)$ such that $P \in \partial \Omega$, the minimizer $f$ of (3) is discontinuous at $P, \partial \Omega \backslash\{P\}$ is smooth $\left(C^{\infty}\right)$, and $f \in C^{0}(\bar{\Omega} \backslash\{P\})$. This is accomplished by replacing $\mathcal{M}$ by a convex set $\mathscr{G}$ such that $\partial \mathscr{G} \backslash\{P\}$ is smooth $\left(C^{\infty}\right)$ and $\mathscr{G} \subset B_{n}\left(O_{n}, p\right)$. We shall use the notation of Section 4.1 throughout this section. We assume $p \in\left(0, \frac{1}{\lambda}\right)$ and set $P=(0, p, 0, \ldots, 0)$. (We will no longer require Figure 8 (right) to hold.) 


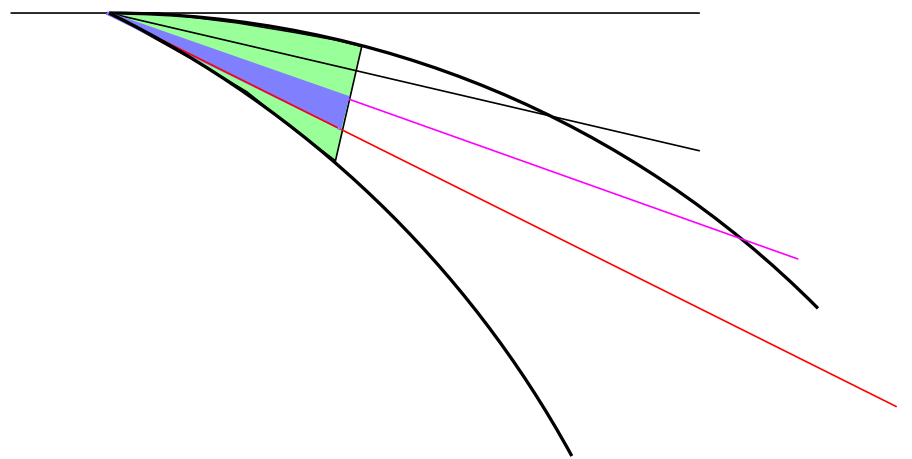

Figure 12. An illustration of $\mathscr{R}_{2}$ (blue region) and $A$ (green and blue regions).
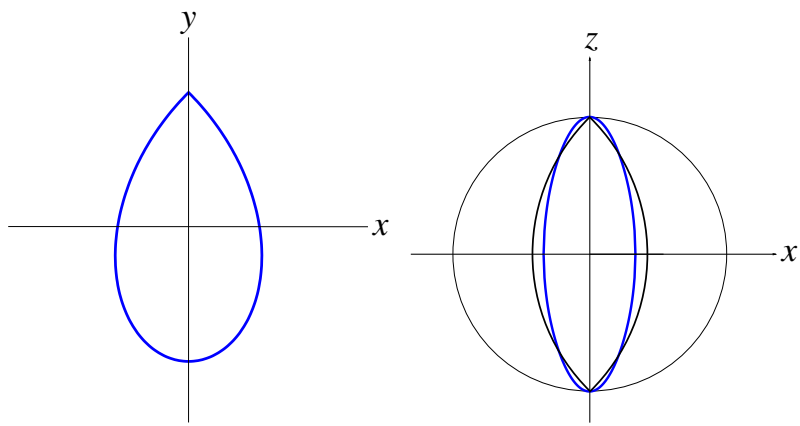

Figure 13. Left: $X\left(\theta, \frac{\pi}{2}, 1\right)$. Right: $X\left(\theta, \frac{1}{2} \arccos (1-\sec (\theta) \sec (2 \theta)), 1\right)$.

Let $\alpha>1, n \geq 3$, and $Y:\left[-\frac{\pi}{2 \alpha}, \frac{\pi}{2 \alpha}\right] \times[0, \pi] \times S^{n-3} \rightarrow \mathbb{R}^{n}$ be defined by

$$
Y(\theta, \phi, \omega)=2 \cos (\alpha \theta) \sin (\phi)(\cos (\theta) \sin (\phi), \sin (\theta) \sin (\phi), \cos (\phi) \omega) .
$$

Let $F: \mathbb{R}^{n} \rightarrow \mathbb{R}^{n}$ be given by

$$
F\left(x_{1}, \ldots, x_{n}\right)=\left(\frac{x_{2}}{p}, \frac{1-x_{1}}{p}, \frac{x_{3}}{p}, \ldots, \frac{x_{n}}{p}\right)
$$

and define $X(\theta, \phi, \omega)=F(Y(\theta, \phi, \omega))$ for $-\frac{\pi}{2 \alpha} \leq \theta \leq \frac{\pi}{2 \alpha}, 0 \leq \phi \leq \pi, \omega \in S^{n-3}$ (see Figures 13 and Figure 14 with $n=3, \alpha=2$; the axes are labeled $x, y, z$ for $x_{1}, x_{2}, x_{3}$, respectively). Let $\mathscr{G}$ be the open, convex set whose boundary is the image of $X$; that is,

$$
\partial \mathscr{G}=\left\{X(\theta, \phi, \omega):-\frac{\pi}{2 \alpha} \leq \theta \leq \frac{\pi}{2 \alpha}, 0 \leq \phi \leq \pi, \omega \in S^{n-3}\right\} .
$$

Notice that $\partial \mathscr{G} \backslash\{P\}$ is a $C^{\infty}$ hypersurface in $\mathbb{R}^{n}$ and $\partial \mathscr{G} \subset \overline{B_{n}\left(\mathcal{O}_{n}, p\right)}$. 
Let $\tau$ satisfy

$$
0<\tau<\min \left\{\frac{p r_{1}}{\sqrt{r_{2}^{2}-r_{1}^{2}}}, \frac{b(4 p-b)}{4(2 p-b)}\right\}
$$

Set $\sigma=-\arctan (\tau / p)$ and $\alpha=\pi /(\pi+2 \sigma)$. Then the tangent cones to $\partial \mathscr{G}$ and $\partial \mathcal{M}$ at $P$ are identical, $\cos (\sigma)>r_{1} / r_{2}$, and (14) holds for $u=\left(u_{1}, u_{2}\right) \in \partial B_{2}\left(O_{2}, p\right)$ with $|u-w|<\delta_{1}$. By making $\tau>0$ smaller if necessary, we may assume $B_{n}\left(\mathcal{O}_{n}, \frac{1}{2}(a+p)-b\right) \subset \mathscr{G}$ if $p<a<p+b$.

Now pick $a \in\left(p, \min \left\{p+b, \frac{1}{\lambda}\right\}\right)$ such that $k_{2}(0, p)-k_{2}(0, a)<-\frac{\beta \sigma}{4}$, as in (30), and define

$$
\Omega=B_{n}\left(O_{n}, a\right) \backslash \bar{G}
$$

Let

$$
m>\max \left\{g_{1}\left(0, r_{1}\right), \frac{1}{2 m_{0}}, \frac{\beta\left(2 \sigma^{2}-\pi\right)}{8 \sigma}\right\}
$$

and define $\phi \in C^{\infty}\left(\mathbb{R}^{n}\right)$ so that $\phi=0$ on $\partial B_{n}\left(\mathbb{O}_{n}, a\right)$ and $\phi=m$ on $\partial \mathscr{G}$, and let $f$ be the variational solution of (1)-(2). Notice that $f \in C^{2}(\Omega)$ satisfies (1) and $f \in C^{0}(\bar{\Omega} \backslash\{P\})$ (e.g., [Lin 1987]).

As in (28), let $B=B_{2}\left(\frac{p-r_{2}}{p} u, r_{2}\right)$. Set $U_{0}=\left\{x \in \Omega: x \in \widetilde{B}, x_{1}>0\right\}$ and $U=\left\{r(\cos (\theta), \sin (\theta) \omega) \in \Omega: r \in(0, p), \theta \in\left(0, \theta_{u}\right), \omega \in S^{n-2}\right\}$. Now $\tilde{g}^{[u]}=0$ on $\partial U_{0} \cap \partial \widetilde{B}$ and $\tilde{g}^{[u]} \leq g_{1}\left(0, r_{1}\right)<m$ on $\partial U_{0} \cap \partial \varphi$ and so Lemma 2, Lemma 3, and (1) imply $\tilde{g}^{[u]} \leq f$ in $U_{0}$ since $f$ minimizes the functional in (3).

As before, set

$$
\begin{aligned}
& \partial_{1} U=\left\{(p \cos (\theta), p \sin (\theta) \omega): \theta \in\left[0, \theta_{u}\right], \omega \in S^{n-2}\right\}, \\
& \partial_{2} U=\partial \mathscr{G} \cap \partial U, \\
& \partial_{3} U=\left\{\left(r \cos \left(\theta_{u}\right), r \sin \left(\theta_{u}\right) \omega\right) \in \bar{\Omega}: r \in[0, p], \omega \in S^{n-2}\right\} .
\end{aligned}
$$

Then $f \geq 0$ on $\partial_{1} U \backslash\{P\}, \partial U=\partial_{1} U \cup \partial_{2} U \cup \partial_{3} U, \tilde{h}_{2} \leq 0 \leq f$ on $\partial_{1} U, \tilde{h}_{2}<m=\phi$ on $\partial_{2} U$, and $\tilde{h}_{2}<\tilde{g}^{[u]}<f$ on $\partial_{3} U$; Lemma 2 implies that (30) continues to hold. Then (24) and (30) imply $f$ is discontinuous at $P$ since $k_{2}(0, p)-k_{2}(0, a)<-\frac{\beta \sigma}{4}$.

\section{The Concus-Finn conjecture}

For the moment, assume $n=2$. Around 1970, Paul Concus and Robert Finn conjectured that if $\kappa \geq 0, \Omega \subset \mathbb{R}^{2}$ has a corner at $P \in \partial \Omega$ of (angular) size $2 \alpha$, $\alpha \in\left(0, \frac{\pi}{2}\right), \gamma: \partial \Omega \backslash\{P\} \rightarrow[0, \pi]$, and $\left|\frac{\pi}{2}-\gamma_{0}\right|>\alpha$, where

$$
\lim _{\partial \Omega \ni x \rightarrow P} \gamma(x)=\gamma_{0}
$$



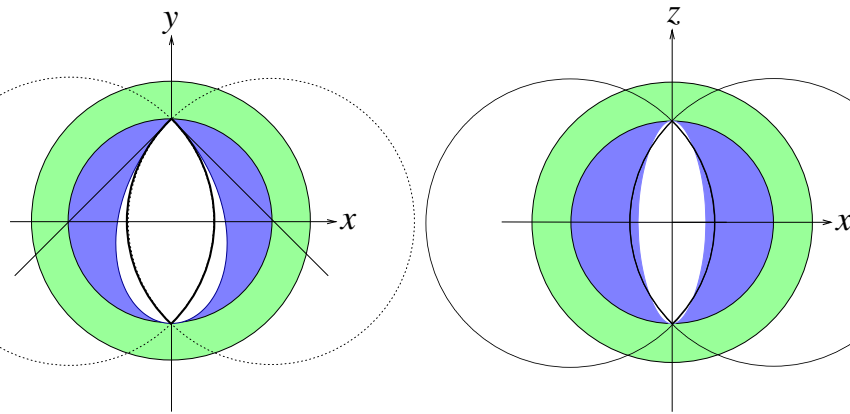

Figure 14. Left: $\Pi_{1,2}(\Omega)$. Right: $\Pi_{1,3}(\Omega)$.

then a function $f \in C^{2}(\Omega) \cap C^{1}(\bar{\Omega} \backslash\{P\})$ which satisfies

$$
\begin{aligned}
\operatorname{div}(T f) & =\kappa f & & \text { in } \Omega, \\
T f \cdot \eta & =\cos (\gamma) & & \text { on } \partial \Omega \backslash\{P\}
\end{aligned}
$$

must be discontinuous at $P$; here $\eta(x)$ is the exterior unit normal to $\Omega$ at $x \in \partial \Omega \backslash\{P\}$.

In the situation above with $\alpha \in\left(\frac{\pi}{2}, \pi\right)$, the "nonconvex Concus-Finn conjecture" states that if $\left|\frac{\pi}{2}-\gamma_{0}\right|>\pi-\alpha$, then the capillary surface $f$ with contact angle $\gamma$ must be discontinuous at $P$. A generalization (including the replacement of (33) by (1)) of this extension of the Concus-Finn conjecture in the case $\gamma_{0} \in(0, \pi)$ was proven in [Lancaster 2012]. Both [Lancaster 2010] and [Lancaster 2012] include the possibility of differing limiting contact angles; that is, the limits

$$
\lim _{\partial^{+} \Omega \ni x \rightarrow P} \gamma(x)=\gamma_{1} \quad \text { and } \quad \lim _{\partial^{-} \Omega \ni x \rightarrow P} \gamma(x)=\gamma_{2}
$$

exist, $\gamma_{1}, \gamma_{2} \in(0, \pi)$, and $\gamma_{1} \neq \gamma_{2}$. Here $\partial^{+} \Omega$ and $\partial^{-} \Omega$ are the two components of $\partial \Omega \backslash\{P, Q\}$, where $Q \in \partial \Omega \backslash\{P\}$. When $\gamma_{1} \neq \gamma_{2}$, the necessary and sufficient (when $\alpha \leq \frac{\pi}{2}$ ) or necessary (when $\alpha>\frac{\pi}{2}$ ) conditions for the continuity of $f$ at $P$ become slightly more complicated.

The cases where $\gamma_{0}=0, \gamma_{0}=\pi, \min \left\{\gamma_{1}, \gamma_{2}\right\}=0$, and $\max \left\{\gamma_{1}, \gamma_{2}\right\}=\pi$ remain unresolved. If we suppose for a moment that the nonconvex Concus-Finn conjecture with limiting contact angles of 0 or $\pi$ is proven, then the discontinuity of $f$ at $P$ in Section 2 follows immediately from the fact that $f<\phi$ in a neighborhood in $\partial \Omega \backslash\{P\}$ of $P$, since then Lemma 1 implies $\gamma_{0}=0$ and therefore $\left|\frac{\pi}{2}-\gamma_{0}\right|>\pi-\alpha$. In this situation (i.e., the solution $f$ of a Dirichlet problem satisfies a 0 (or $\pi$ ) contact angle boundary condition near $P$ ), establishing the discontinuity of $f$ at $P$ would be much easier and a much larger class of domains $\Omega$ with a nonconvex corner (i.e., $\alpha>\frac{\pi}{2}$ ) at $P$ would have this property. For example, if $\Omega$ is a bounded locally Lipschitz domain in $\mathbb{R}^{2}$ for which (4) holds, $f \in C^{2}(\Omega)$ is a generalized solution of (1)-(2) (and $H$ need not vanish), and $\phi$ is large enough near $P$ (depending 
on $H$ and the maximum of $\phi$ outside some neighborhood of $P$ ) that $f<\phi$ on $\partial \Omega \backslash\{P\}$ near $P$, then the fact that $\gamma_{0}=0$ (Lemma 1) together with the nonconvex Concus-Finn conjecture would imply that $f$ is discontinuous at $P$.

Now consider $n \in \mathbb{N}$ with $n \geq 3$. Formulating generalizations of the Concus-Finn conjecture in the "convex corner case" (i.e., $\Omega \cap B_{n}(P, r) \subset\left\{X \in \mathbb{R}^{n}:(X-P) \cdot \mu>0\right\}$ for some $\mu \in S^{n-1}, P \in \partial \Omega$ and $\left.r>0\right)$ and in other cases where $\partial \Omega$ is not smooth at a point $P \in \partial \Omega$ may be complicated because the geometry of $\partial \Omega \backslash\{P\}$ is much more interesting when $n>2$. Establishing the validity of a generalization of the Concus-Finn conjecture for solutions of (1) and (34) when $n>2$ is probably significantly harder than doing so when $n=2$.

Suppose we knew that a solution $f$ of (1) and (34) is necessarily discontinuous at a "nonconvex corner" $P \in \partial \Omega$ when $\gamma_{0}=0$, where $\gamma_{0}$ is given by (32). In this case, a necessary condition for the continuity of $f$ at $P$ would be that

$$
\begin{aligned}
& \limsup _{\partial \Omega \ni X \rightarrow P} T f(X) \cdot \eta(X)>0, \\
& \liminf _{\partial \Omega \ni X \rightarrow P} T f(X) \cdot \eta(X)<\pi .
\end{aligned}
$$

Then the arguments in Section 4 could be made more easily and the conclusion that $f$ is discontinuous at $P$ would hold in a much larger class of domains $\Omega$; here, of course, we use the ridge point $P$ in Section 4 as an example of a "nonconvex corner" of a domain in $\mathbb{R}^{n}$. The primary difficulty in proving in Section 4 that $f$ is discontinuous at $P$ is establishing (30); a more "natural" generalization of $\Omega \subset \mathbb{R}^{2}$ in Section 2 would be

$$
\Omega^{*}=\left\{\left(x \omega_{1}, y, \omega_{2}, \ldots, \omega_{n-1}\right) \in \mathbb{R}^{n}:(x, y) \in B_{2}\left(\mathcal{O}_{2}, a\right) \backslash \bar{M}, \omega \in S^{n-1}\right\} .
$$

However, the use of Lemma 3 to help establish (30) in $\Omega^{*}$ is highly problematic. On the other hand, an $n$-dimensional "Concus-Finn theorem" for a nonconvex conical point (e.g., $P \in \partial \Omega^{*}$ ) would only require an inequality like (26) to prove that $f<\phi$ on $\partial \Omega \backslash\{P\}$ near $P$, and hence that $f$ is discontinuous at $P$; the replacement of (17) by (31) in order to obtain $\Omega$ such that $\partial \Omega \backslash\{P\}$ is $C^{\infty}$ would be unnecessary.

\section{References}

[Bourni 2011] T. Bourni, " $C^{1, \alpha}$ theory for the prescribed mean curvature equation with Dirichlet data”, J. Geom. Anal. 21:4 (2011), 982-1035. MR 2836589 Zbl 1232.53009

[do Carmo and Dajczer 1982] M. P. do Carmo and M. Dajczer, "Helicoidal surfaces with constant mean curvature", Tôhoku Math. J. (2) 34:3 (1982), 425-435. MR 84f:53003 Zbl 0501.53003

[Elcrat and Lancaster 1986] A. R. Elcrat and K. E. Lancaster, "Boundary behavior of a nonparametric surface of prescribed mean curvature near a reentrant corner", Trans. Amer. Math. Soc. 297:2 (1986), 645-650. MR 87h:35098 Zbl 0602.35042

[Finn 1986] R. Finn, Equilibrium capillary surfaces, Grundlehren der Mathematischen Wissenschaften 284, Springer, New York, 1986. MR 88f:49001 Zbl 0583.35002 
[Gerhardt 1974] C. Gerhardt, "Existence, regularity, and boundary behavior of generalized surfaces of prescribed mean curvature", Math. Z. 139 (1974), 173-198. MR 55 \#10846 Zbl 0316.49005

[Giusti 1976] E. Giusti, "Boundary value problems for non-parametric surfaces of prescribed mean curvature", Ann. Scuola Norm. Sup. Pisa Cl. Sci. (4) 3:3 (1976), 501-548. MR 58 \#2572 Zbl 0344.35036

[Giusti 1978] E. Giusti, "On the equation of surfaces of prescribed mean curvature: Existence and uniqueness without boundary conditions”, Invent. Math. 46:2 (1978), 111-137. MR 58 \#7337 Zbl 0381.35035

[Huff and McCuan 2006] R. Huff and J. McCuan, "Scherk-type capillary graphs", J. Math. Fluid Mech. 8:1 (2006), 99-119. MR 2006k:76022 Zbl 1125.76012

[Huff and McCuan 2009] R. Huff and J. McCuan, "Minimal graphs with discontinuous boundary values", J. Aust. Math. Soc. 86:1 (2009), 75-95. MR 2010k:35203 Zbl 1161.53010

[Jenkins and Serrin 1968] H. Jenkins and J. Serrin, "The Dirichlet problem for the minimal surface equation in higher dimensions", J. Reine Angew. Math. 229 (1968), 170-187. MR 36 \#5519 Zbl 0159.40204

[Korevaar 1980] N. J. Korevaar, "On the behavior of a capillary surface at a re-entrant corner”, Pacific J. Math. 88:2 (1980), 379-385. MR 82c:49033 Zbl 0475.49034

[Korevaar and Simon 1996] N. Korevaar and L. Simon, "Equations of mean curvature type with contact angle boundary conditions", pp. 175-201 in Geometric analysis and the calculus of variations, edited by J. Jost, Int. Press, Cambridge, MA, 1996. MR 98e:35071 Zbl 0932.35091

[Lancaster 1985] K. E. Lancaster, "Boundary behavior of a nonparametric minimal surface in $\mathbf{R}^{3}$ at a nonconvex point”, Analysis 5:1-2 (1985), 61-69. MR 86m:49053 Zbl 0601.35035

[Lancaster 1988] K. E. Lancaster, "Nonparametric minimal surfaces in $\mathbf{R}^{3}$ whose boundaries have a jump discontinuity", Internat. J. Math. Math. Sci. 11:4 (1988), 651-656. MR 89m:35072 Zbl 0696.35057

[Lancaster 2010] K. E. Lancaster, "A proof of the Concus-Finn conjecture", Pacific J. Math. 247:1 (2010), 75-108. MR 2011m:53011 Zbl 1256.53009

[Lancaster 2012] K. E. Lancaster, "Remarks on the behavior of nonparametric capillary surfaces at corners”, Pacific J. Math. 258:2 (2012), 369-392. MR 2981959 Zbl 1253.35069

[Lancaster and Siegel 1996] K. E. Lancaster and D. Siegel, "Existence and behavior of the radial limits of a bounded capillary surface at a corner", Pacific J. Math. 176:1 (1996), 165-194. MR 98g:58030a Zbl 0866.76018

[Lau and Lin 1985] C. P. Lau and F.-H. Lin, "The best Hölder exponent for solutions of the nonparametric least area problem”, Indiana Univ. Math. J. 34:4 (1985), 809-813. MR 87d:49058 Zbl 0581.49025

[Lin 1987] F.-H. Lin, "Behaviour of nonparametric solutions and free boundary regularity", pp. 96-116 in Miniconference on geometry and partial differential equations, 2 (Canberra, 1986), edited by J. E. Hutchinson and L. M. Simon, Proc. Centre Math. Anal. Austral. Nat. Univ. 12, Austral. Nat. Univ., Canberra, 1987. MR 89c:35047 Zbl 0647.35032

[Melin 2013] J. Melin, Examples of discontinuity for the variational solution of the minimal surface equation with Dirichlet data on a domain with a nonconvex corner and locally negative mean curvature, MS Thesis, Wichita State University, 2013, Available at http://hdl.handle.net/10057/ 10639.

[Nitsche 1989] J. C. C. Nitsche, Lectures on minimal surfaces, I: Introduction, fundamentals, geometry and basic boundary value problems, Cambridge Univ. Press, 1989. MR 90m:49031 Zbl 0688.53001 
[Serrin 1969] J. Serrin, "The problem of Dirichlet for quasilinear elliptic differential equations with many independent variables", Philos. Trans. Roy. Soc. London Ser. A 264 (1969), 413-496. MR 43 \#7772 Zbl 0181.38003

[Shi and Finn 2004] D. Shi and R. Finn, "On a theorem of Lancaster and Siegel”, Pacific J. Math. 213:1 (2004), 111-119. MR 2004m:76038 Zbl 1156.76361

[Simon 1976] L. Simon, "Boundary regularity for solutions of the non-parametric least area problem", Ann. of Math. (2) 103:3 (1976), 429-455. MR 58 \#30681 Zbl 0335.49031

[Williams 1978] G. H. Williams, "Surfaces of prescribed mean curvature with inequalities on the boundary”, Math. Z. 164:1 (1978), 31-51. MR 80d:53006 Zbl 0378.49029

Received November 3, 2014. Revised March 21, 2015.

KIRK E. LANCASTER

DePartment of MATHEMATICS, STATistics, AND Physics

WiChita STATE UNIVERSITY

WICHITA, KS 67260-0033

UNITED STATES

lancaster@math.wichita.edu

JARON MELIN

Department of Mathematics, Statistics, and Physics

WICHITA STATE UNIVERSITY

WICHITA, KS 67260-0033

UNITED STATES

melin@math.wichita.edu 


\title{
PACIFIC JOURNAL OF MATHEMATICS
}

\author{
msp.org/pjm
}

Founded in 1951 by E. F. Beckenbach (1906-1982) and F. Wolf (1904-1989)

\section{EDITORS}

Don Blasius (Managing Editor)

Department of Mathematics

University of California

Los Angeles, CA 90095-1555

blasius@math.ucla.edu

\author{
Paul Balmer \\ Department of Mathematics \\ University of California \\ Los Angeles, CA 90095-1555 \\ balmer@math.ucla.edu \\ Robert Finn \\ Department of Mathematics \\ Stanford University \\ Stanford, CA 94305-2125 \\ finn@math.stanford.edu \\ Sorin Popa \\ Department of Mathematics \\ University of California \\ Los Angeles, CA 90095-1555 \\ popa@math.ucla.edu
}

\author{
Vyjayanthi Chari \\ Department of Mathematics \\ University of California \\ Riverside, CA 92521-0135 \\ chari@math.ucr.edu \\ Kefeng Liu \\ Department of Mathematics \\ University of California \\ Los Angeles, CA 90095-1555 \\ liu@math.ucla.edu \\ Jie Qing \\ Department of Mathematics \\ University of California \\ Santa Cruz, CA 95064 \\ qing@ cats.ucsc.edu
}

\section{PRODUCTION}

Silvio Levy, Scientific Editor, production@msp.org

\section{SUPPORTING INSTITUTIONS}

ACADEMIA SINICA, TAIPEI

CALIFORNIA INST. OF TECHNOLOGY

INST. DE MATEMÁTICA PURA E APLICADA

KEIO UNIVERSITY

MATH. SCIENCES RESEARCH INSTITUTE

NEW MEXICO STATE UNIV.

OREGON STATE UNIV.

\author{
STANFORD UNIVERSITY \\ UNIV. OF BRITISH COLUMBIA \\ UNIV. OF CALIFORNIA, BERKELEY \\ UNIV. OF CALIFORNIA, DAVIS \\ UNIV. OF CALIFORNIA, LOS ANGELES \\ UNIV. OF CALIFORNIA, RIVERSIDE \\ UNIV. OF CALIFORNIA, SAN DIEGO \\ UNIV. OF CALIF., SANTA BARBARA
}

\author{
Daryl Cooper \\ Department of Mathematics \\ University of California \\ Santa Barbara, CA 93106-3080 \\ cooper@math.ucsb.edu \\ Jiang-Hua Lu \\ Department of Mathematics \\ The University of Hong Kong \\ Pokfulam Rd., Hong Kong \\ jhlu@maths.hku.hk \\ Paul Yang \\ Department of Mathematics \\ Princeton University \\ Princeton NJ 08544-1000 \\ yang@math.princeton.edu
}

These supporting institutions contribute to the cost of publication of this Journal, but they are not owners or publishers and have no responsibility for its contents or policies.

See inside back cover or msp.org/pjm for submission instructions.

The subscription price for 2016 is US $\$ 440 /$ year for the electronic version, and $\$ 600 /$ year for print and electronic.

Subscriptions, requests for back issues and changes of subscriber address should be sent to Pacific Journal of Mathematics, P.O. Box 4163, Berkeley, CA 94704-0163, U.S.A. The Pacific Journal of Mathematics is indexed by Mathematical Reviews, Zentralblatt MATH, PASCAL CNRS Index, Referativnyi Zhurnal, Current Mathematical Publications and Web of Knowledge (Science Citation Index).

The Pacific Journal of Mathematics (ISSN 0030-8730) at the University of California, c/o Department of Mathematics, 798 Evans Hall \#3840, Berkeley, CA 94720-3840, is published twelve times a year. Periodical rate postage paid at Berkeley, CA 94704, and additional mailing offices. POSTMASTER: send address changes to Pacific Journal of Mathematics, P.O. Box 4163, Berkeley, CA 94704-0163.

PJM peer review and production are managed by EditFLOW ${ }^{\circledR}$ from Mathematical Sciences Publishers.

\section{PUBLISHED BY}

\section{mathematical sciences publishers \\ nonprofit scientific publishing}

http://msp.org/

(C) 2016 Mathematical Sciences Publishers 


\section{PACIFIC JOURNAL OF MATHEMATICS}

Volume $282 \quad$ No. $2 \quad$ June 2016

Exhausting curve complexes by finite rigid sets

JAVIER ARAMAYONA and CHRISTOPHER J. LEININGER

A variational characterization of flat spaces in dimension three

Giovanni Catino, Paolo Mastrolia and Dario D. Monticelli

Estimates of the gaps between consecutive eigenvalues of Laplacian

293

DAgUang Chen, TAO Zheng and HongCANG YANG

Liouville type theorems for the $p$-harmonic functions on certain manifolds

JINGYI CHEN and YUE WANG

Cartan-Fubini type rigidity of double covering morphisms of quadratic manifolds

\section{HOSUNG KIM}

On the uniform squeezing property of bounded convex domains in $\mathbb{C}^{n}$

KANG-TAE KIM and LIYOU ZHANG

Lefschetz pencils and finitely presented groups

RYOMA KOBAYASHI and NAOYUKI MONDEN

Knot homotopy in subspaces of the 3-sphere

YUYA KODA and MAKOTO OzAWA

On the relationship of continuity and boundary regularity in prescribed mean curvature Dirichlet problems

KIRK E. LANCASTER and JARON MELIN

Bridge spheres for the unknot are topologically minimal

JUNG HOON LEE

On the geometric construction of cohomology classes for cocompact discrete subgroups of $\mathrm{SL}_{n}(\mathbb{R})$ and $\mathrm{SL}_{n}(\mathbb{C})$

SUSANNE SCHIMPF

On Blaschke's conjecture

Xiaole Su, Hongwei Sun and Yusheng Wang

The role of the Jacobi identity in solving the Maurer-Cartan structure equation 